\title{
Anti-TNF and Crohn's Disease: When Should We Stop?
}

\author{
Edouard Louis, Jacques Belaiche and Catherine Reenaers \\ Dept. of gastroenterology CHU Liege, and GIGA Research University of Liege, Belgium
}

\begin{abstract}
When to stop anti-TNF therapy in Crohn's disease (CD)? This is a very important question both for patients and physicians. There is no published evidence to clearly and definitely answer this question. However data on natural history of $\mathrm{CD}$, long term safety of biologies, outcome after immunosuppressors (IS) cessation and some preliminary studies on biologies cessation may help us to discuss this topic. One could argue that there is currently no good reason to stop anti-TNF therapy in a patient who is in stable remission and can tolerate this drug very well. The decision to stop an anti-TNF treatment is thus currently based on a compromise between the benefits/risks and cost of such long term treatment. While it appears now clearly that prolonged anti-TNF therapy is associated with favourable outcome with sustained remission, reduced surgeries and hospitalisation as well as absence of significant increase in mortality or cancers, the cost-effectiveness which is probably favourable for short and mid-term treatment (up to one year), may be less optimal for very long term treatment. In this perspective however, prospective studies should be performed to adequately assess long term evolution, disease outcome, safety and global cost of strategies based on treatment reduction with IS maintenance alone or even full treatment cessation.
\end{abstract}

Key Words: Infliximab, adalimumab, certolizumab pegol, Crohn's disease, therapy, biomarkers, mucosal healing.

\section{INTRODUCTION}

The question of anti-TNF treatment duration in CD is certainly a very important one and is one of the greatest preoccupations of patients. When starting a new treatment in $\mathrm{CD}$, one of the first questions the patients may have is usually: "when will I be able to stop this treatment"? While the question is very relevant, we actually have very little data to give a clear answer, because the controlled data we have with pivotal trials usually give us efficacy data for remission and response induction and for remission and response maintenance over a one year period [1-3].

However we have most often indirect elements to help us and discuss this point of optimal duration of anti-TNF treatment in CD: these elements are the natural history of the disease, the available data with immunosuppressive drugs, the long term safety of biologies, and a few investigator-initiated studies having started to address this question. Beyond that, the cessation of a biological treatment in IBD must be decided on a case-by-case basis and adapted strategies must be proposed. The cost of such long term treatment may also become an important issue. While we now tend to use anti-TNF earlier in the disease and in a larger proportion of the patients, the cost-effectiveness of such strategies over the very long term must be questioned.

\section{NATURAL HISTORY OF CD}

$\mathrm{CD}$ is considered as a chronic relapsing disease, but in a large proportion of patients, it is actually a chronic active disease. Population-based and cohort studies have showed that only a small half of the patients have little evolutive disease with low prevalence of relapses, hospitalizations, or complications [4-7]. These patients probably do not need anti-TNF therapy and if such treatment has been used, an arrest must certainly be discussed as soon as the flare has been controlled. The other patients will develop complications including strictures and internal or perianal fistulas over the course of the disease $[8,9]$. These will lead to hospitalizations and surgeries and will considerably interfere with patients every day life and long term projects. For these patients a sustained control of the disease process is strongly warranted and an effective treatment can only be stopped if reasonable evidence shows absence of activity of this process. There is probably a difference between early disease and long-lasting disease, the reversal and control of the disease process being more difficult and unstable in the latter situation. Long-lasting disease are indeed characterized by anatomical damages including mucosal and submucosal architectural changes, fibrosis, strictures and complex fistulas that will favour clinical relapse and 
that will render a deep and stable remission more difficult to achieve. Furthermore, immunological status of the patients may change over the course of the disease [10]. In parallel, immunisation against luminal material may increase, enhancing the potential reactivation of the immune process $[11,12,13]$. As a correlate, a stable remission will usually be more difficult to obtain in long-lasting diseases [14] and these diseases will usually be more treatment-dependent. This certainly represents an argument for earlier treatment with anti-TNF in CD. These patients being treated earlier with anti-TNF and in whom a more complete reversal of the immune process and the tissue lesions can be achieved are also probably better candidates for treatment cessation.

\section{OUTCOME AFTER IMMUNOSUPPRESSOR DISCONTINUATION}

Although immunosuppressive drugs are globally less effective than anti-TNF in CD, inducing less clinical remission and less mucosal healing [15], the outcome after discontinuation of these drugs may help to imagine what would happen after anti-TNF cessation. In an observational GETAID study of relapse after azathioprine cessation in $\mathrm{CD}$, it was shown that the longer the duration of remission under azatioprine, the lowest the risk of relapse was [16]. Particularly, the risk of relapse seemed particularly low after 4 years of sustained remission. The same group then embarked a placebo-controlled trial of azathioprine prolonged treatment beyond 42 months of sustained remission [17]. This trial showed that the strategy of treatment cessation was not equivalent to treatment prolongation. Particularly, 18-months relapse rate were around $20 \%$ in the cessation group as compared to $10 \%$ in the prolongation group. Over a longer follow-up period, the relapse rate in the azathioprine cessation group continued to increase up to $60 \%$ after 3 years [18]. This study indicates that even in patients in stable remission, the cessation of an immunosuppressive drug is associated with an increased risk of relapse. The risk of relapse in the cessation group remains however reasonably low over a one year period and one could consider drug cessation for a given period of time, on a case-by-case basis after discussion with the patients. No such data are available with methotrexate in CD. The big difference between immunosuppressor and anti-TNF cessation is that in the former situation, no active drug is left to treat the patient, while in the case of anti-TNF discontinuation an immunosuppressive drug can be given as maintenance therapy.

\section{LONG TERM SAFETY OF ANTI-TNF}

While short and mid term safety and tolerance of anti-TNF is usually very good, the fear of long term complication is generally the reason why both patients and physicians would like to stop the drug when the disease has been completely stabilized. This fear is based on the mechanism of action of these drugs. They block the tumor necrosis factor alpha which is a pivotal cytokine in some anti-microbial and anti-tumoral physiological processes. Indeed, the best documented side-effects are the increased risk of tuberculosis [19] and of other infections, mainly with intra-cellular pathogens (mycobacteria, lysteria, histoplasmosis...), as well as a probable slight increase in the risk of lymphoma [20]. The active and systematic search for latent tuberculosis has already significantly decreased the incidence of active tuberculosis under anti-TNF treatment. Furthermore, a recent meeting of the European Crohn and Colitis Organisation on infections and biologies has proposed a series of guidelines, including vaccination against herpes zoster, hepatitis $\mathrm{B}$, influenza and streptococcus pneumoniae as well as avoiding some aliments potentially containing germs as lysteria (i.e. unpasteurized milk or insufficiently cooked meat) [21]. These guidelines should also in a near future help and diminish fatal complications linked to biologies. Another measure that gains more and more support is the avoidance of long term combined treatment with immunosuppressors. While over the short term and in immu-nosuppressor-naïve patients a combination therapy revealed superior to either immunosuppressor or anti-TNF monotherapies [15], there is currently very little evidence for a cumulative benefit of these drugs over a longer term or in immunosuppressor failures [1, 2 , 22]. On the other hand, combined therapies were associated in a retrospective study with a very significant increase in the risk of opportunistic infections with a relative risk of 12 when two treatments were combined [23]. It is of note however that in this study many opportunistic infections recorded were very benign and that it is mmainly the combination of immunosuppressor or anti-TNF with steroids which was associated with such infection while there were too few patients under immunosuppressor and anti-TNF combination to draw any conclusion on this type of co-treatment. Nevertheless, in the reported pediatric series of nearly universally fatal hepato-splenic T cell lymphoma in infliximab treated patients, all the patients affected had been treated with combined therapy with thiopurines [24]. All together, these measures should lower the risk profile associated with biologies and allow the physician to prescribe them for enough time to achieve stable and durable remission of the disease.

\section{AVAILABLE STUDIES ON ANTI-TNF TREATMENT CESSATION IN IBD}

Early data with infliximab in CD were only short term induction data [25]. Only one single infusion was used at that time and it is striking to note that some patients had a very prolonged clinical response or even remission 
after such isolated infusion [26]. These data already suggested that prolonged treatment was probably not necessary in all the treated patients. Since then however, it has become clear that such one-shot treatment was not a good option for the majority of patients because the median time to relapse was 10 weeks and because reuse of infliximab more than 4 months after a single infusion was associated with high risk of allergic reaction. More recently the "bridge" study of the GETAID explored the idea of a 3-dose infliximab induction given in parallel with immunosuppressor that would then maintain the remission [27]. The results of this study were rather disappointing. While the short term effect of infliximab was very strong, the maintenance effect with immunosuppressor was globally rather weak. After one year, the overall sustained remission rate in these patients was low and actually close to the one of patients receiving a placebo induction. Only in the patients who were immunosuppressor-nai've at the time of infliximab induction, the benefit was more consistent with a reasonable $40 \%$ remission rate after one year. This study clearly shows that in patients who have failed under immunosuppressors, a longer period of anti-TNF treatment is necessary to obtain a durable remission and allow treatment cessation. Long term results of the large Leuven cohort of patients treated with infliximab indicate that in real life, a significant proportion of patients (around 20\%) are in sustained remission despite infliximab cessation [28]. This possibility has been prospectively explored in a recent GETAID study, only published in abstract form [29]. In this prospective cohort study, 115 patients with a stable remission on combined immunosuppressor-infliximab therapy for more than one year had their infliximab stopped, while pursuing immunosuppressive treatment. After one year more than half of the patients were still in sustained remission. Factors associated with a low risk of relapse included low CDAI, low highly sensitive CRP, low fecal calprotectin, high haemoglobin levels and non smoking. These features indicate that the patients with a low risk of relapse are the ones who are in very deep not only clinical, but also endoscopic and biological remission. Another important question to address when contemplating anti-TNF discontinuation, is the possibility to restart this treatment in case of relapse. Here also the data of the GETAID study may be reassuring, at least over the short term, since almost all the relapsers who were retreated were in remission again 4 weeks after first retreatment.

\section{COST-EFFECTIVENESS}

Cost studies in CD have essentially included direct medical costs. First studies were based on models generated with data form the literature [30]. These studies indicated that hospitalisations and surgeries represented the majority of the direct medical costs (up to $80 \%$ ) linked to CD. They were further confirmed by several retrospective and prospective cohort analyses [31,32], suggesting that a therapeutic strategy that would decrease the number of hospitalisations and/or surgeries in CD might have an important impact on direct medical costs. Prospective studies with both infliximab and adalimumab given for induction of remission and then maintenance treatment have showed a significant decrease in both hospitalisations and surgeries with these drugs [33, 34]. Globally, this may suggest a potential cost-effectiveness of anti-TNF at least over one year of treatment, although no proper cost-effectiveness analysis has been fully published so far. However, beyond one year of treatment, the cost-effectiveness of continuous scheduled anti-TNF treatment would require a major superiority of this strategy as compared to maintenance with immunosuppressive treatment and/or on demand anti-TNF. In view of the preliminary results of the GETAID infliximab discontinuation study mentioned here above [29], this result may be difficult to reach, at least in patients in long lasting stable remission.

\section{PRACTICAL CONSIDERATIONS FOR STOPPING ANTI-TNF TREATMENT IN CD}

Usually, a biological treatment is started in patients who do no longer respond to conventional therapies. In patients responding to the treatment, it is certainly not wise to contemplate a treatment cessation as long as a complete clinical remission has not been achieved. In patients who have been in clinical remission for a sufficient period of time, it is probably useful to assess biological as well as endoscopic signs of disease activity. In several models and clinical situations, C-reactive protein (CRP) serum concentration has been associated with the risk of relapse [35]. More sophisticated serum or stool markers have also been proposed, but their added value as compared to CRP has not been clearly demonstrated [36-38]. A stable value of CRP within normal range should therefore also be obtained before anti-TNF cessation. The recent data from the GETAID study may even indicate that hsCRP should be preferred to classical CRP in assessing such patients, since mild variations within the normal range of classical CRP may have clinical significance to disclose deeper remission [29]. Correlation between clinical indexes of activity or biological markers of inflammation and mucosal healing is not very strong [39]. Mucosal healing under anti-TNF treatment has been associated with a decrease in relapse rate, hospitalisation and surgeries [31]. A third condition for biologies cessation is thus to control for a mucosal healing in patients with ileo-colonic disease accessible to endoscopic exploration. For patients with proximal small bowel disease, there is not universally accepted exploration to assess the control of inflammation at the tissue level. However, entero-MRI could be a good candidate [40]. An absence of mucosal lesion (not always 
easy to detect), and of contrast enhancement of the bowel wall or the mesenterium could be interpreted as tissue healing. Globally, fecal calprotectin is now considered as a relatively good surrogate marker for mucosal healing [38, 41-42]. However, this correlation has been shown to be stronger for ulcerative colitis than for CD. Furthermore fecal calprotectin appears to be essentially correlated with colonic mucosal lesions while the correlation with small bowel lesion is much weaker. Nevertheless, in the above-mentioned GETAID study [29], fecal calprotectin was in univariate analysis the factor most strongly associated with the time-to-relapse. It is however too early to say that, at least for colonic disease, it could replace endoscopy for patient's assessment.

\section{CONCLUSIONS}

Globally, the decision to stop or carry on with anti-TNF treatment in CD is based on an estimated benefit-risk ratio and on the cost-effectiveness of long term treatment. The patient must certainly be fully and clearly informed on both advantages and risks linked to any therapeutic strategy that is proposed. In patients with unstable chronic active disease, stopping an effective treatment will put the patients at risk of worsening and complications development and should probably not be attempted. However in patients stabilized for a reasonably long period of time, a careful assessment of the clinical, biological and endoscopic situation may help to take a thoughtful decision in collaboration with the patient himself. From the payer's point of view, this may allow to keep some resources for giving the possibility of early anti-TNF treatment in adequately selected patients.

\section{REFERENCES}

[1] Hanauer S, Feagan B, Lichtenstein G, Mayer LF, Schreiber S, Colombel JF, et al. Maintenance infliximab for Crohn's disease: the ACCENT I randomized trial. Lancet 2002; 359: 1541-9.

[2] Colombel J, Sandborn W, Rutgeerts P, Enns R, Hanauer SB, Panaccione R, et al. Adalimumab for maintenance of clinical response and remission in patients with Crohn's disease: the CHARM trial. Gastroenterology 2007; 132: 52-65.

[3] Schreiber S, Khaliq-Kareemi, Lauwrance I, Thomsen 00, Hanauer SB, McColm J, et al. Maintenance therapy with certolizumab pegol for Crohn's disease. N Engl J Med 2007; 357: 239-50

[4] Munkholm P, Langholz E, Davidsen M, Binder V. Disease activity courses in a regional cohort of Crohn's disease patients. Scand J Gastroenterol 1995; 30: 699-706.

[5] Wolters FL, Russel MG, Sijbrandij J, Ambergen T, Odes S, Rhs L, et al. Phenotype at diagnosis predicts recurrence rates in Crohn's disease. Gut 2006; 55: 1124-30.

[6] Beaugerie L, Seksik P, Nion-Larmurier I, Gendre JP, Cosnes J. Predictors of Crohn's disease. Gastroenterology 2006; 130 : 650-6.

[7] Loly C, Belaiche J, Louis E. Predictors of severe Crohn's disease. Scand J Gastroenterol 2008; 43 : $948-54$.

[8] Louis E, Collard A, Oger AF, Degroote E, Aboul Nasr El Yafi FA, Belaiche J. Behaviour of Crohn's disease according to the Vienna Classification: changing pattern over the course of the disease. Gut 2001; 49: 777-82.

[9] Cosnes J, Cattan S, Blain A, Beaugerie L, Carbonnel F, Pare R, et al. Long- term evolution of disease behaviour of Crohn's disease. Inflamm Bowel Dis 2002;8:244-50.

[10] Kugathasan S, Saubermann L, Smith L, Kou D, Itoh J, Binion DG, et al. Mucosal T-cell immunoregulation varies in early and late inflammatory bowel disease. Gut 2007; 56: 1696-1705.

[11] Dubmsky M, Kugathasan S, Mei L, Picornell Y, Nebel J, Wrobel IT, et al. Increased immune reactivity predicts aggressive complicating Crohn's disease in Children. Clin Gastroenterol Hepatol 2008; 6: 1105-11.

[12] Rieder F, Schleder S, Wolf A, Dirmeier A, Strauch U, Obermeier F, et al. Specific levels and combinations of the anti-glycan antibodies anti-L, anti-C, ALCA, ACCA, gASCA and AMCA contribute to diagnosis and differential diagnosis of patients with Crohn's disease and are associated with complicated disease and surgery. Gastroenterology 2008; 134(A-53): 392.

[13] Ferrante M, Henckaerts L, Joossens M, Pierik M, Joossens S, Dotan N, et al. New serological markers in inflammatory bowel disease are associated with complicated disease behaviour. Gut 2007; 56: 1394-403.

[14] Schreiber S, Hanauer S, Lichtenstein G, Sandborn W. Superior efficacy of certolizumab pegol in early Crohn's disease is independent of CRP status. Gastroenterology 2007; 132(A-510): T1298. 
[15] Colombel JF, Rutgeerts P, Reinisch W, Mantzaris GJ, Kornbluth A, Rachmilewitz D, et al. A randomized, double-blind, controlled trial comparing infliximab and infliximab plus azathioprine to azathioprine in patients with Crohn's disease naive to immunomodulators and biologic therapy. Gut 2008; 57(sП): Al.

[16] Bouhnik Y, Lemann M, Mary JY, Scemama G, Tai R, Matuchansky C, et al. Long-term follow-up of patients with Crohn's disease treated with azathioprine or 6-mercaptopurine. Lancet 1996; 347: 215-9.

[17] Lemann M, Mary JY, Colombel JF, Duclos B, Soule JC, Lerebours E, et al. A randomized, double-blind, controlled withdrawal trial in Crohn's disease patients in long-term remission on azathioprine. Gastroenterology 2005; 128: 1812-08.

[18] Treton X, Bouhnik Y, Mary JY, Colombel JF, Duclos B, Soule JC, et al. Azathioprine withdrawal in patients with Crohn's disease maintained on prolonged remission: a high risk of relapse. Clin Gastroenterol Hepatol 2009; $7:$ 80-5.

[19] Keane J, Gershon S, Wise R, Mirabile-Levens E, Kasznica J, Schwieterman WD, et al. Tuberculosis associated with infliximab, a tumor necrosis factor alpha-neutralizing agent. N Engl J Med 2001; 345: 1098-104.

[20] Siegel C, Sadie M, Marden S, Persing SM, Larson RJ, Sands BE. Risk of lymphoma associated with anti-TNF agents for the treatment of Crohn's disease: a meta-analysis. Gastroenterology 2008; 134(A-144): 970.

[21] Rahier JF, Ben-Horm S, Chowers Y, Conlon C, De Munter P, D'Haens G, et al. European evidence-based consensus on the prevention, diagnosis and management of opportunistic infections in inflammatory bowel disease. J Crohn's colitis 2009; 3: 47-91.

[22] Van Assche G, Magdelaine-Beuzelin C, D'Haens G, Baert F, Noman M, Vermeire S, et al. Withdrawal of immunosuppression in Crohn's disease treated with scheduled infliximab maintenance: a randomized trial. Gastroenterology 2008; 134: 1861-8.

[23] Toruner M, Loftus E, Harmsen W, Zinsmeister AR, Orenstein R, Sandborn WJ, et al. Risk factors for opportunistic infections in patients with inflammatory bowel disease. Gastroenterology 2008; 134: 929-36.

[24] Mackey A, Green L, Liang L, Dinndorf P, Avigan M. Hepatosplenic T cell lymphoma associated with infliximab use in young patients treated for inflammatory bowel disease. J Pediatr Gastroenterol Nutr 2007; 44: 265-7.

[25] Targan S, Hanauer S, Van Deventer S, Mayer L, Present DH, Braakman T, et al. A short-term study of chimeric monoclonal antibody cA2 to tumor necrosis factor alpha for Crohn's disease. Crohn's Disease cA2 Study Group. NEngl J Med 1997; 337: 1029-35.

[26] Rutgeerts P, D'Haens G, Targan S, Vasiliauskas E, Hanauer SB, Present DH, et al. Efficacy and safety of retreatment with antitumor necrosis factor antibody (infliximab) to maintain remission in Crohn's disease. Gastroenterology 1999; 117: 761-9.

[27] Lemann M, Mary JY, Duclos B, Veyrac M, Dupas JL, Delchier JC, et al. Infliximab plus azathioprine for steroid-dependent Crohn's disease patients: a randomized placebo-controlled trial. Gastroenterology 2006; 130: 1054-61.

[28] Schnitzler F, Fidder H, Ferrante M, Noman M, Arijs I, Van Assche G, et al. Long-term outcome of treatment with infliximab in 614 patients with Crohn's disease: results from a single-centre cohort. Gut 2009; 58: 492-500.

[29] Louis E, Vernier-Massouille G, Grimaud J, Bouhnik Y, Laharie D, Dupas JL, et al. Infliximab discontinuation in Crohn's disease patients in stable remission of combined therapy with immunosuppressors : interim analysis of a prospective cohort study. Gut 2008 ; 57 (s1l): A66.

[30] Hay JW, Hay AR. Inflammatory bowel disease: medical cost algorithms. J Clin Gastroenterol 1992; 14: 318-27.

[31] Feagan BG, Vreelad MG, Larson LR, Bala MV. Annual cost of care for Crohn's disease: a payer perspective. Am J Gastroenterol 2000; 95: 1955-60.

[32] Silverstem MD, Loftus EV, Sandborn WJ, Tremame WJ, Feagan BG, Nietert PJ, et al. Clinical course and costs of care for Crohn's disease: Markov model analysis of a population-based cohort. Gastroenterology 1999; 117: 49-57.

[33] Rutgeerts P, Feagan BG, Lichtenstein GR, Mayer LF, Schreiber S, Colombel JF, et al. Comparison of scheduled and episodic treatment strategies of infliximab in Crohn's disease. Gastroenterology 2004; 126: 402-13.

[34] Loftus EV, Feagan BG, Colombel JF, Rubm DT, Wu EQ, Yu AP, et al. Effects of adalimumab maintenance therapy on healthrelated quality of life of patients with Crohn's disease: patient-reported outcomes of the CHARM trial. Am J Gastroenterol 2008; 103: $3132-$ 41.

[35] Vermeire S, Van Assche G, Rutgeerts P. C-reactive protein as a marker for inflammatory bowel disease. Inflamm Bowel Dis 2004; 10: 661-5.

[36] Louis E, Belaiche J, Van Kemseke C, De Groote D, Geenen V, Mary JY. Concentration of interleukin-6 in the serum predicts relapse in Crohn's disease. Eur J Gastroenterol Hepatol 1997; 9: 939-44. 
Published in : Current Drug Targets (2009)

Status: Postprint (Author's version)

[37] Louis E, Belaiche J, Van Kemseke C, Schaaf N, Mahieu P. Soluble interleukin-2 receptor in Crohn's disease: disease activity assessment and prediction of relapse. Dig Dis Sci 1995; 40: 1750-6.

[38] Costa F, Mumolo M, Ceccarelli L, Bellini M, Romano MR, Sterpi C, et al. Calprotectin is a stronger predictive marker of relapse in ulcerative colitis than in Crohn's disease. Gut 2005; 54: 364-8.

[39] Cellier C, Sahmoud T, Froguel E, Adenis A, Belaiche J, Bretagne JF, et al. Correlations between clinical activity, endoscopic severity, and biological parameters in colonic or ileocolonic Crohn's disease. A prospective multicenter study of 121 cases. Gut $1994 ; 35: 231-5$.

[40] Ryan E, Heaslip I. Magnetic resonance enteroclysis compared with conventional enteroclysis and computed tomography enteroclysis: a critically appraised topic. Abdom Imaging 2008; 33: 34-7.

[41] Jones J, Loftus EV Jr, Panaccione R, Chen LS, Peterson S, McConnell J, et al. Relationships between disease activity and serum and fecal biomarkers in patients with Crohn's disease. Clin Gastroenterol Hepatol 2008; 6: 1218-24.

[42] Sipponen T, Savilahti E, Karkkainen P, Kolho KL, Nuutinen H, Turunen U, et al. Fecal calprotectin, lactoferrin, and endoscopic disease activity in ,monitoring anti-TNF-alpha therapy for Crohn's disease. Inflamm Bowel Dis 2008; 14: 1392-8. 